\title{
A baseline estimate of population size for monitoring the Endangered Madagascar giant jumping rat Hypogeomys antimena
}

\author{
Richard P. Young, Anselme Toto Volahy, Robert Bourou, Richard Lewis, \\ Joanna Durbin, Tim J. Wright, Tim D. Hounsome and Julia E. Fa
}

\begin{abstract}
The Endangered Madagascar giant jumping rat, Hypogeomys antimena, has suffered a major decline in distribution and is now restricted to two seemingly unconnected sub-populations in the largest remaining fragment of deciduous, seasonally dry forest in Menabe, western Madagascar. In a previous study a rapid decrease in numbers of $H$. antimena was observed in relatively intact forest, suggesting a factor of population decline additional to habitat loss, and provoking fears of a negative trend occurring across its remaining range. In the current study we conducted extensive line transect surveys to estimate active $H$. antimena burrow density in 2004 and 2005 as an index of population size, and trapping to estimate mean group size, as a multiplier for population size estimation. Within the surveyed areas we estimated the combined size of the two $H$. antimena subpopulations in 2005 to be c. 36,000 , considerably larger than previously assumed. There was no evidence that active burrow density across the species' known range changed between 2000 and 2005. H. antimena was not uniformly distributed, with higher densities of active burrows found in forest with the highest canopy in areas furthest from forest edges. These core forest areas are vital for the species' conservation and the recent declaration that the Menabe forest will receive statutory protection provides hope that $H$. antimena may be safeguarded. However, given its restricted range and low reproductive output, among other factors, $H$. antimena remains threatened and requires close future monitoring.
\end{abstract}

Keywords Distance sampling, dry forest, habitat fragmentation, Madagascar, population monitoring, status survey.

Richard P. Young* (Corresponding author), Tim J. Wright and Julia E. FA Durrell Wildlife Conservation Trust, Les Augrès Manor, Trinity, Jersey, JE3 5BP, UK Channel Islands. E-mail richard.young@durrell.org

Anselme Toto Volahy, Robert Bourou, Richard Lewis and Joanna DuRBIN Durrell Wildlife Conservation Trust-Madagascar, BP8511, Antananarivo 101, Madagascar.

Tim D. Hounsome Biocensus, 3 All Saints Fields, Summer Street, Stroud, Gloucestershire, GL5 1NE, UK.

*Also at: Department of Biology \& Biochemistry, University of Bath, Bath, BA2 7AY, UK.

Received 25 April 2007. Revision requested 11 June 2007.

Accepted 30 August 2007.

Article updated on 12 August 2022

\section{Introduction}

0 eciduous, seasonally dry forest in western Madagascar harbours high numbers of animal and plant endemics but has been dramatically reduced in extent and become highly fragmented through encroachment by subsistence agriculture and timber harvesting. This habitat is now one of the highest conservation priorities in Madagascar (Ganzhorn et al., 2001). Dry forest once covered much of western Madagascar but by 1990 only an estimated c. $3 \%$ of the original cover remained (Smith, 1997). As a result, many species associated with this habitat have suffered concomitant declines in their distribution and population size and are now lost or threatened with extinction.

The Menabe region of central western Madagascar, between the Tsiribihina and Tomitsy rivers, contains one of the largest remaining tracts of deciduous dry forest. Until recently the Menabe forest was largely unprotected and over 1995-2000 c. 4\% was being lost per annum through clearfelling and burning, or was heavily degraded through human disturbance, including timber extraction (Sommer et al., 2002). However, since 2000, local and international conservation partners have intensified measures to arrest deforestation. In 2006 the forest was given protected area status by the Madagascar Government as a move towards implementing a long-term management plan for biodiversity conservation and sustainable use of the area's natural resources.

The Madagascar giant jumping rat Hypogeomys antimena is a large rodent, endemic to the Menabe forest (Cook et al., 1991) and considered a flagship species for the dry forest habitat. It has probably one of the most restricted ranges of any Malagasy mammal (Goodman \& Rakotondravony, 1996) and is categorized as Endangered on the IUCN Red List (IUCN, 2007). The extent of occurrence of the species was estimated to have declined by $52 \%$ over $1985-2000$ (Sommer et al., 2002) and H. antimena is now thought to be limited to two of the least modified blocks of the Menabe forest, known as the Kirindy/CFPF and Ambadira forests, which are c. $200 \mathrm{~km}^{2}$ in area (Sommer \& Tichy, 1999; Sommer \& Hommen, 2000). A global H. antimena population of c. 9,000 adults was estimated from a 2000-2001 survey (Sommer et al., 2002). Based on these data a population viability analysis predicted that if past deforestation rates continue the species would become extinct within 24 years (Sommer et al., 2002). 
Observations from an intensive study of $H$. antimena in the Kirindy/CFPF forest suggested that the species may be declining even in relatively undisturbed forest (Sommer \& Hommen, 2000). Between 1992 and 2000 the study population fell by c. $55 \%$ (Sommer \& Hommen, 2000). Because $H$. antimena is an obligate monogamous species, with a low reproductive output of 1-2 offspring per year (Sommer, 2001), these declines were perceived not as part of a population cycle but of a long-term negative trend that may be occurring across its range (Sommer \& Hommen, 2000). The main proposed explanation was predation by domestic dogs belonging to tenrec (Tenrecidae) hunters (Sommer \& Hommen, 2000), although disease was also suspected.

However, the decline reported in H. antimena (Sommer et al., 2002) was largely based on one study site, and may not therefore be representative of the entire population. To determine more accurately the current size of the $H$. antimena population and any short-term trends, we carried out extensive line transect surveys across its known range in 2004 and 2005. We present the results of generalized linear models to assess the relationship between general habitat characteristics and the distribution and numbers of the species. We also make recommendations for the design of an ongoing monitoring programme for $H$. antimena.

\section{Study area}

The deciduous dry forests of Menabe are characterized by the presence of baobab trees (Adansonia spp.) and occur primarily on sandy and lateritic soils. The region's climate is dominated by a dry season (April-November), with a hot, wet season in the intervening period. Average total annual rainfall is c. $800 \mathrm{~mm}$ (Sorg \& Rohner, 1996).

$H$. antimena is thought to occur in two sub-populations, one each in the two main blocks of the Menabe forest, the Ambadira forest in the north and the Kirindy/CFPF forest in the south (Sommer \& Tichy, 1999; Fig. 1). From surveys conducted by Sommer et al. (2002) in 2000, the northern and southern sub-populations were estimated to be distributed over c. 4,000 and 15,000 ha, respectively, of suitable habitat. These surveys revealed that $H$. antimena had probably disappeared from dry forest to the west of the main road that runs south to north through the Menabe region, and from the forest corridor that connects the Ambadira and Kirindy/CFPF forests (Fig. 1). They also indicated that $H$. antimena is absent from areas of forest near to permanent rivers, presumably because burrows would be flooded during the wet season (Toto Volahy, pers. comm.). There is reasonably strong evidence (Toto Volahy, 1999; Sommer et al., 2002) that H. antimena does not occur in forest that is substantially modified through human activity, and disappears quickly following disturbance. To design the survey we inspected a 2003 Landsat 7 satellite image and, in combination with available species distribution information, we delineated areas of potentially suitable habitat. We estimated there is 17,202 ha of potentially suitable habitat in the Ambadira forest and 15,599 ha in Kirindy/CFPF forest, and these areas of forest were defined, for the purposes of this study, as the northern and southern survey areas respectively (Fig. 1). Because of a lack of field data during the design phase the delineation of the survey areas was subjective, and therefore it is possible that we did not cover the entire range of $H$. antimena.

\section{Methods}

\section{Estimation of active burrow density}

H. antimena live together as adult pairs with one or two young, and use underground burrows to rest during the day and to avoid predation (Cook et al., 1991). Regularly used burrows (hereafter referred to as active) are clearly identifiable from inactive ones by the presence of recently dug soil and often by the absence of leaves, twigs or cobwebs in the burrow entrance. Recently dug soil is distinctive as it differs in colour and structure to the surface soil. An intensive mark-recapture study (Sommer \& Hommen, 2000) indicated that family groups use only one burrow for extensive periods of time, and thus the density of active burrows can be used as an index of population size of $H$. antimena (Sommer et al., 2002).

A plot sampling survey of active burrows was previously carried out during April-November in 2000 and 2001 (Sommer et al., 2002). Each plot was $1 \mathrm{~km}^{2}$ in area and was surveyed by teams of five people walking systematically across the plot and counting and marking all active and inactive burrows observed (as active burrows are relatively easily detected in the forest during the dry season it was considered likely that few were missed). Four and six plots were surveyed in the northern and southern study areas, respectively. Full details of the survey design and data collection protocols are provided in Sommer et al. (2002). Seven of these 10 plots fell within the area of the 2004-2005 survey and these data were used to estimate retrospectively mean active burrow density for 2000-2001. The remaining three plots, in which no active burrows were recorded in 2000-2001, fell outside the 2004-2005 survey area and were therefore not included in the present analysis.

From 8 July to 30 October 2004 and 21 July to 19 October 2005 line transect surveys of $H$. antimena burrows were undertaken in the northern and southern study areas (see Fig. 1 for survey design). The majority of fieldwork in 2004 and 2005, as well as in 2000-2001, was carried out by A. Toto Volahy. A total of $152.5 \mathrm{~km}$ line transects, each arranged in a rectangle $(1.00 \times 0.25 \mathrm{~km}$, hereafter referred to as a rectangular shaped transect) were randomly positioned in the northern survey area, and 19 rectangular shaped transects in the south. Transects were rectangular 


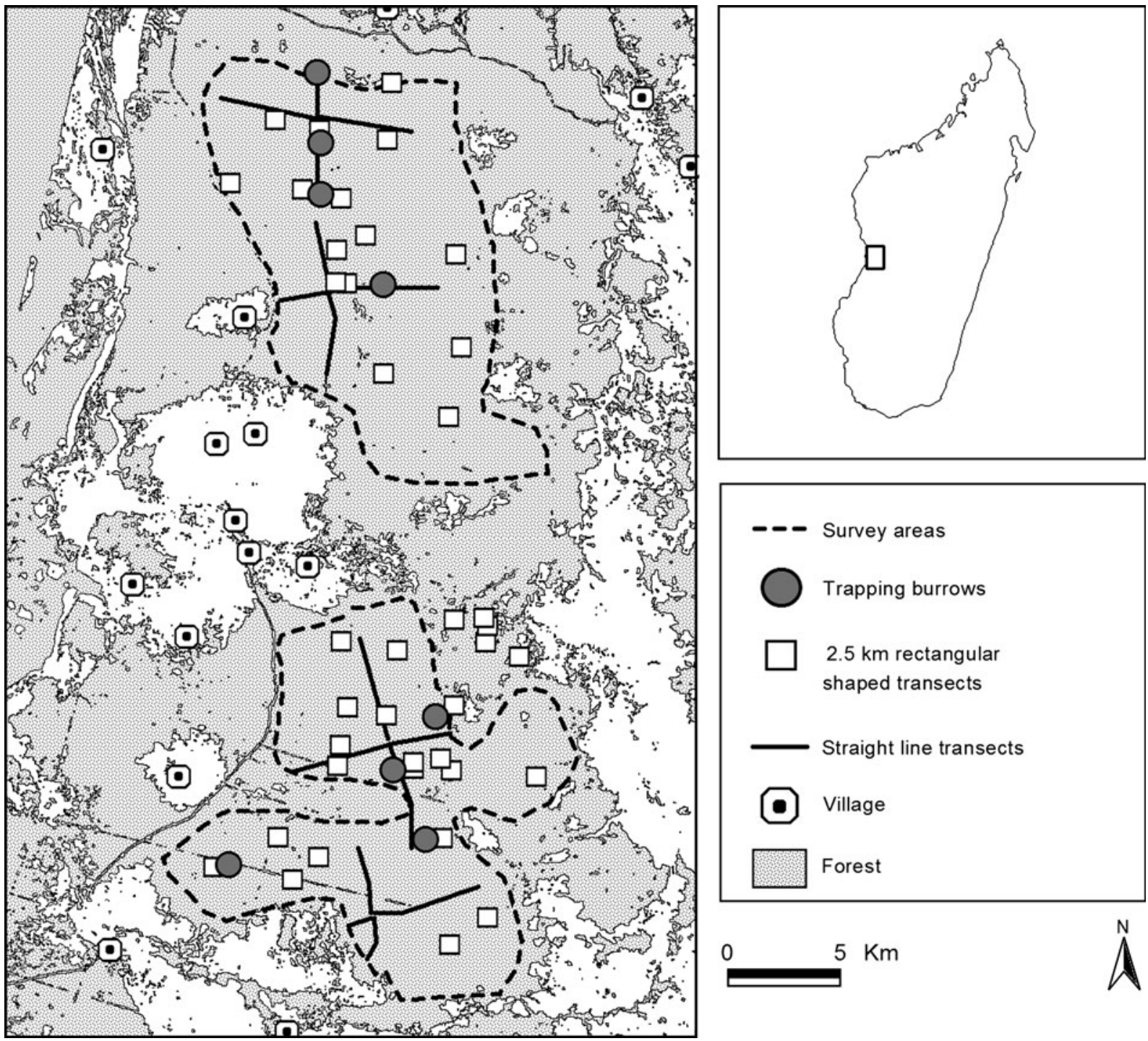

FIG. 1 Survey design showing the locations of line transects, and active burrows where trapping was carried out, in the Menabe forest in central western Madagascar. The map of the forest is drawn from a Landsat 7 image acquired in 2003. Inset indicates the position of the main map in western Madagascar.

(with the longest sides along a north-south axis) to allow fieldworkers to finish in the same location as the starting point, to make travelling between transects more efficient. A further eight straight line transects $(2-5 \mathrm{~km}$ long) were surveyed in the north and eight straight line transects (2-5 km long) in the south. The straight line transects were arranged in groups of four running approximately in a north, east, south and west direction from a randomly placed starting point for $5 \mathrm{~km}$ or until the edge of the survey area was reached (following the approach used by Sommer et al., 2002). The same rectangular shaped and straight line transects were surveyed in both 2004 and 2005, and in total $137.9 \mathrm{~km}$ of line transects were surveyed annually. A further $172.5 \mathrm{~km}$ rectangular shaped transects, randomly placed in both northern and southern survey areas, were surveyed in 2004 and pooled with the other 2004 data to investigate habitat associations of $\mathrm{H}$. antimena. A further five $2.5 \mathrm{~km}$ rectangular shaped transects were placed at random within the corridor region between the northern and southern survey areas (see Fig. 1), and were surveyed in 2004. Distance sampling (Buckland et al., 2001) was used to estimate the detection probability of active and inactive burrows.

Line transects were walked at c. $2-3 \mathrm{~km}$ per hour, following a pre-determined compass bearing; the distance walked was measured with a hip-chain (Forestry Suppliers, Jackson, USA). When a burrow was observed, the fieldworker walked a short distance past the burrow following the bearing on the compass before removing the hip-chain and placing it on the ground, so that the exact position of the line transect was clearly marked, and then measured the perpendicular distance from the line transect to the burrow. 
Because the diameter of burrow systems are wide relative to the distance at which burrows tended to be observed, the perpendicular distances between the line transect and the holes in the burrow system nearest and furthest from the transect were measured, with the mean of these two values used as the perpendicular distance. If the line transect ran through the burrow system the distances to the holes furthest from the line on either side of the transect were measured, the smallest value assigned a negative value, and the mean of the two values was the perpendicular distance.

\section{Habitat characteristics}

Habitat variables were measured at four points located halfway along each side of the rectangular transects. Within a $10 \mathrm{~m}$ radius of each point we quantified: (1) number of trees (plants $>8 \mathrm{~m}$ in height), (2) estimated height and measured diameter at breast height (dbh) of each tree, (3) number of bushes (plants $<8 \mathrm{~m}$ height), (4) estimated height and measured dbh of each bush, (5) canopy cover (estimated visually to nearest $10 \%$ ), and (6) visibility (average of maximum visible distance in the direction of the four main points of a compass). The mean height and diameters of trees and bushes within the $10 \mathrm{~m}$ radius was then calculated for each sample point.

\section{Estimation of mean group size}

In July-August 2005 a trapping study to estimate mean group size was conducted at eight active burrow systems, randomly selected from all known burrows identified in 2004 and during the first part of the 2005 survey. Each burrow system was trapped for three consecutive nights with unbaited Tomahawk traps $(48.3 \times 16.5 \times 16.5 \mathrm{~cm}$; Tomahawk, Wisconsin, USA) at the entrance to each hole in the burrow system, and dead branches placed around the traps to ensure animals could not escape capture when leaving the burrow. Traps were set at c. 15.00 and checked hourly from 18.00 until 04.00 (Sommer \& Hommen, 2000), after which they were removed. Regular checking of the traps was important to minimize the risk of interference by fossa Cryptoprocta ferox, a predator of $H$. antimena.

Animals caught were placed in cloth bags. Following Sommer (1997) captured animals were sexed and weighed to estimate age (juvenile $250-500 \mathrm{~g}$, subadult 500-900 g, and adult $>900 \mathrm{~g})$. They were permanently marked with passive integrated transponder tags (ZooChip, AEG, Germany) and also temporarily identified with livestock dye so that recaptured animals could be easily detected on subsequent nights and not handled again. Animals were released at the entrance of the burrow in which they were caught once trapping had ceased and the traps removed.

\section{Data analyses}

Active and inactive burrow density was estimated using the software Distance v. 4.o (Thomas et al., 2003). Suitable models recommended by Buckland et al. (2001) were considered, and detection probability histogram and goodness of fit test statistics examined. On the basis of the lowest Akaike's Information Criterion value, the uniform cosine model was chosen to fit the detection functions for both active and inactive burrows. The 2004 and 2005 data were pooled to produce a global detection function but density estimation was post-stratified by year. A modified version of a two-tailed $z$-test (Buckland et al., 2001) was used (to allow for the non-independence of density estimates resulting from the use of a global detection function) to test the difference between the density estimates of active burrows in 2004 and 2005. Density estimation was then post-stratified by year and region to provide active burrow density estimates for the northern and southern subpopulations in 2005. The mean and standard error of group size, estimated from the trapping study, were entered as generic multipliers in the Distance analysis to estimate total population size in 2005 .

The minimum extent of occurrence of $H$. antimena in the northern and southern areas was estimated using a geographical information system (ArcView v. 3.2, ESRI, Redlands, USA) to draw a minimum convex polygon around the locations of all active burrows identified in the 2005 line transect survey for both northern and southern survey areas.

A generalized linear model (GLM) was used to investigate associations between habitat variables and the occurrence (detection/non-detection) and count of active burrows along the $2.5 \mathrm{~km}$ line transects. Encounter rate (burrows $\mathrm{km}^{-1}$ ) was not used as a response variable in this analysis as it was not normally distributed and did not appear to fit any other statistical distribution. Habitat data collected at systematic sample points were averaged to produce a single figure for each line transect. The occurrence of active burrows was modelled with a binomial distribution and logit link function and the count of active burrows with a Poisson distribution and logarithm link function. Forward and backward stepwise regression was used to select explanatory variables for inclusion in the models. Only significant explanatory variables $(\mathrm{P}<0.05)$ were retained in the model. Additionally, a binomial logistic and Poisson regression were used to investigate the relationship between the occurrence and count of burrows respectively, and distance to nearest forest edge. The latter explanatory variable was calculated in ArcView by measuring from the centre point of the line transect to the nearest edge of the main contiguous forest blocks. The relationship between habitat variables and distance to forest edge was investigated by means of a linear regression. 
All statistical analyses were conducted using GenStat v. 6.2 (Lawes Agricultural Trust, Rothamstead, UK).

\section{Results}

\section{Temporal changes in active burrow density}

The density of active burrows in 2000-2001 was $27.7 \mathrm{~km}^{-2}$ (Sommer et al., 2002; Table 1). During the line transect surveys in 2004 and 2005, 49 and 78 active burrows were observed, respectively. Active burrows were typically detected to a distance of $\mathrm{c} .11 \mathrm{~m}$ from the transect line but the probability of their detection declined markedly at distances $>_{4} \mathrm{~m}$. Mean density of active burrows in 2004 was estimated to be $27.8 \mathrm{~km}^{-2}$ (Table 1), almost identical to the $2000-$ 2001 density estimate. The number of active burrows recorded in 2005 was much higher, despite the same survey effort, at 40.4 burrows $\mathrm{km}^{-2}$ (Table 1), i.e. an increase of $45.3 \%$. However, there was no evidence that the overall population changed in density between these years $(z=-1.647$, $\mathrm{P}=0.100$ ). The estimates of density of active and inactive burrows combined declined by $3.9 \%$ between 2004 and 2005 as a result of a fall of $28.6 \%$ in the mean density of inactive burrows in 2005 (Fig. 2). Although active burrow density was similar in 2000-2001 and 2004, inactive burrow density was c. $20 \%$ lower in the former period (Fig. 2).

\section{Estimated population size}

From the 2005 trapping study we estimated mean group size to be $2.75 \pm$ SE 0.31 (range 2-4). All but one group contained an adult pair and six groups appeared to include offspring, which were probably born in 2005 or 2004. Including the estimate of the mean and standard error of group size as multipliers in the Distance analysis, we estimated the combined size of the two sub-populations to be 36,476 (95\% confidence interval, CI, 24,838-53,568) in 2005. The uncertainty in the population estimate comes from the detection probability $(9.8 \%)$, encounter rate $(57.2 \%)$ and multiplier (33.0\%). Assuming only two adults per active burrow (following Sommer et al., 2002), we estimated the

TABLE 1 Estimates of mean $H$. antimena active burrow density $\left(\mathrm{km}^{-2}\right)$, with \% coefficient of variation (CV) and 95\% confidence interval (CI) from a plot sampling survey in 2000-2001 (Sommer et al., 2002) and line transect surveys in 2004 and 2005. The location of sampling units in 2000-2001 differs from 2004 and 2005 .

\begin{tabular}{llll}
\hline & $\begin{array}{l}\text { Mean active burrow } \\
\text { density }\left(\mathrm{km}^{-2}\right)\end{array}$ & \% CV & 95\% CI \\
\hline Year & 27.7 & 21.7 & $15.9-39.5$ \\
$2000-2001$ & 27.8 & 17.9 & $19.5-39.7$ \\
2005 & 40.4 & 16.1 & $29.4-55.6$ \\
\hline
\end{tabular}

size of the northern and southern sub-populations of adults to be 18,040 (95\% CI 12,204-26,666) and 9,168 (95\% CI 5,63014,932) respectively, in 2005.

\section{Spatial patterns and habitat associations}

Before the line transect surveys were conducted the area of potentially suitable habitat in the northern and southern study areas was subjectively estimated to be 17,202 and 15,599 ha, respectively. However, H. antimena were not uniformly distributed within these areas and appeared to be absent from some parts (Fig. 3). From the minimum convex polygons we estimated the minimum extents of occurrence of the southern and northern sub-population to be 9,889 and 8,910 ha, respectively. No active or inactive burrows were recorded along the five transects positioned in the forest corridor region between the two survey areas.

Line transects with the highest encounter rates of active burrows tended to be found in the centre of the northern and southern study areas (Fig. 3). The number of active burrows recorded on line transects was positively associated with distance to the edge of the forest blocks (GLM: $F_{1,49}=14.96$, $\mathrm{P}<0.001$ ) as was the occurrence of active burrows (GLM: $\left.F_{1,49}=19.81, \mathrm{P}<0.001\right)$. A GLM predicted that, on average, the probability of the presence of active burrows increases to $>90 \%$ in areas that are $>5 \mathrm{~km}$ from the edge of the main forest blocks, and that there is at least a $50 \%$ probability of active burrows occurring in areas $>3 \mathrm{~km}$ from the forest edge.

Both the occurrence (GLM: $F_{1,49}=12.55, \mathrm{P}<0.001$ ) and numbers (GLM: $F_{1,49}=13.70, \mathrm{P}<0.001$ ) of active burrows were positively associated with the mean height of trees. However, there was no evidence that active burrow occurrence and numbers were related to density of trees and bushes, dbh of trees and bushes, canopy cover, or visibility. The GLM of active burrow occurrence predicted, on average, a probability $>50 \%$ of active burrows occurring in areas where mean canopy height is $\geq 11.5 \mathrm{~m}$. The probability of occurrence of active burrows was predicted to decline below $20 \%$ if mean canopy height was $<10 \mathrm{~m}$.

Mean canopy height increased with distance to forest edge (mean canopy height $=10.10+0.0003 \times$ distance to forest edge; $F_{1,49}=12.43, \mathrm{P}<0.001, r^{2}=0.186$ ), although this variable only explained $19 \%$ of the variation in mean canopy height.

\section{Discussion}

Further to historical and large-scale population decline through habitat loss, a recent study (Sommer \& Hommen, 2000) revealed that $H$. antimena rapidly decreased in abundance in at least one part of its remaining range and in relatively undisturbed dry forest. This led to a suspicion that a factor other than habitat loss was causing the decline 


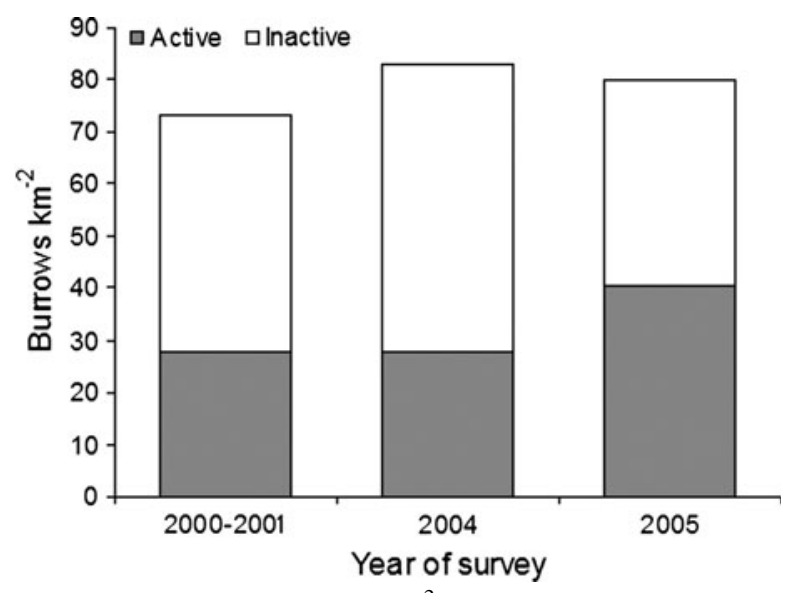

FIG. 2 Mean density (burrows $\mathrm{km}^{-2}$ ) of active and inactive burrows in 2000-2001 (Sommer et al., 2002), 2004 and 2005.

and that this negative trend may be occurring across its entire range (Sommer \& Hommen, 2000; Sommer et al., 2002). Our current study, however, demonstrates $H$. antimena is considerably more abundant than was previously assumed and provides evidence that overall its population has not declined in density since 2000. It has also shown that the northern sub-population was approximately an order of magnitude larger (18,0oo adults) than the previous estimate (1,800 adults, Sommer et al., 2002), and twice the size of the southern sub-population, and therefore clearly far more important than formerly thought.

Although there was a marked increase in the estimates of active burrow density between 2004 and 2005, there was no statistical evidence of a change in the overall population during this time period. An increase in active burrow density of $45 \%$ in one year was unexpected, given that $H$. antimena produce only 1-2 offspring per year (Sommer, 2001). However, overall burrow density remained largely unchanged between 2004 and 2005 but the proportion of active burrows increased and consequently inactive burrows decreased. This pattern is consistent with a growing population, as $H$. antimena tend to colonize inactive burrows rather than dig new burrows (Sommer \& Hommen, 2000). The relatively low estimate of inactive burrow density from plot sampling in 2000-2001 (Sommer et al., 2002) probably resulted from the lack of an estimate of detection probability and the difficulty of detecting inactive burrows that are located more than c. $5 \mathrm{~m}$ from the observer. Further monitoring is needed to confirm the possibility of population growth and to investigate long-term population trends. We advocate use of line transect sampling for future monitoring as it provides an estimate of detection probability and is relatively simple to implement. We also recommend the survey design developed in this study because it is efficient in terms of the number of field-days required to generate reliable burrow density estimates of reasonable precision.
However, there are two potential problems with using active burrows for monitoring $H$. antimena. Firstly, misclassification of active burrows would lead to bias in the population estimate. Based on the combination of evidence from previous studies (Toto Volahy, 1999; Sommer \& Hommen, 2000; Sommer et al., 2002) and data presented here, the signs of recent digging at burrows and the lack of debris in the tunnel entrances provide a clear indication of activity. Any change in protocol for active burrow classification over time may have confounded our assessment of population change but the majority of data collection in 2000-2001, 2004 and 2005 was carried out by one experienced person. Nevertheless, if a monitoring programme for $H$. antimena based on burrows is to be implemented by the future protected area management authority, more work is needed to develop and test a standardized protocol for classifying activity that will be robust to inter-observer differences and usable by less experienced personnel.

Secondly, numbers of active burrows only provide an index of abundance as these may not be related linearly to population size. For example, $H$. antimena exhibit two phases of dispersal, at the end of the dry and wet season, when male and female offspring, respectively, tend to disperse from their natal territory (Sommer, 1996). The colonization of new burrows by dispersing offspring would increase active burrow density, even though population size would remain relatively constant. However, all but one of the eight groups trapped in 2005 contained at least one adult male and female, which would suggest that increased colonization of burrows by juveniles in 2005 does not explain the observed rise in the estimates of active burrow density. Furthermore, we aimed to avoid phases of dispersal by surveying between July and October and carrying out surveys at the same time of year in 2004 and 2005 to prevent seasonal effects confounding population estimates. The estimate of group size was based on a relatively small number of samples $(\mathrm{n}=8)$ and trapping at more burrows would have increased our confidence in this estimate and the precision of the overall population size estimate. To increase sample size substantially, considerable resources would be needed because of the labour-intensiveness of trapping and the logistics of following a randomized sampling design. Nevertheless, given its potential impact on the accuracy of population size estimation we recommend trapping to estimate group size be incorporated into future population monitoring.

The range of the northern and southern sub-populations of $H$. antimena in 2000-2001 was reported to be c. 4,000 and 15,000 ha, respectively (Sommer et al., 2002) but we estimated the species' minimum extent of occurrence in 2004 to be c. 9,000 ha and 10,000 ha, respectively. The northern and southern sub-populations therefore appear more and less widely distributed, respectively, than hitherto assumed. However, because our classification of potentially suitable 

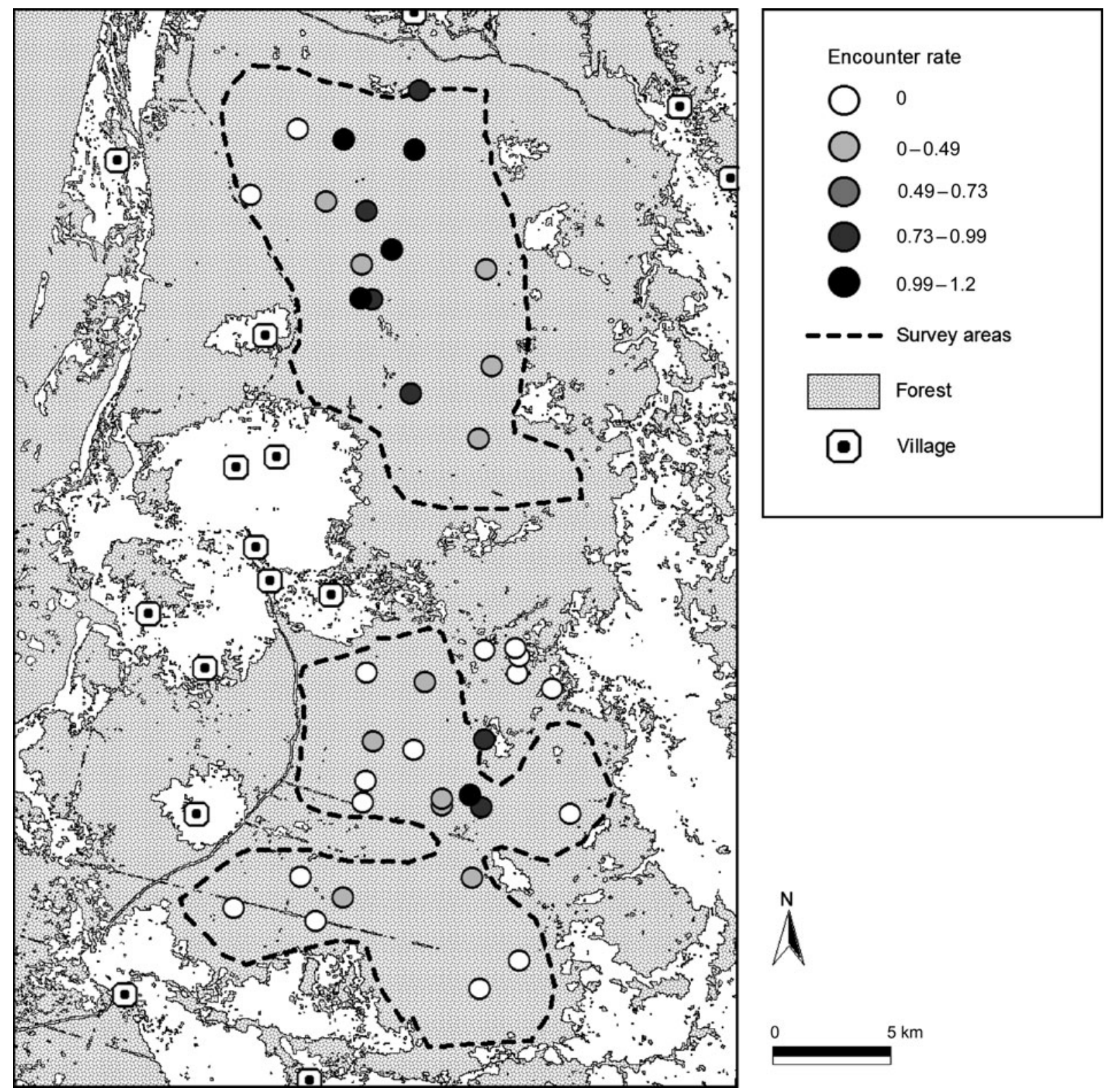

FIG. 3 Map of variation in encounter rate of H. antimena (active burrows $\mathrm{km}^{-1}$ ) along $2.5 \mathrm{~km}$ line transects in the survey areas in 2005 and in the corridor region in 2004.

habitat was subjective our surveys may have missed parts of the species' range and therefore $H$. antimena may be more widely distributed and abundant than the current estimates. Given the apparent specific habitat requirements of $H$. antimena for relatively undisturbed dry forest (Toto Volahy, 1999; Sommer et al., 2002) we consider it unlikely that large parts of the species' range were missed as our survey covered the majority of remaining intact habitat. The absence of active or inactive burrows in the forest corridor between the northern and southern survey areas indicates that the two sub-populations are not connected. However, by chance, the five transects placed in the corridor were clustered and therefore did not provide good spatial coverage. Further surveying and a better understanding of the habitat associations of $H$. antimena is needed to map the limits of its distribution accurately.

Our data have demonstrated that the probability of occurrence and numbers of active burrows were positively related to distance to the edge of the main forest blocks. Active burrows were found at highest densities in the core areas of both the the Kirindy/CFPF and Ambadira forests, and were often absent at the periphery. Distance to forest edge was positively correlated with canopy height, which in turn was also associated with active burrow occurrence and numbers. Forest with high canopy is likely to have been subject to less timber extraction, which tends to target the most mature trees, and therefore can be considered to be the least modified. The mechanism responsible for this pattern, 
however, is unclear; reduced food availability, predation by feral dogs or a shift in predator-prey dynamics in modified habitats are hypotheses that warrant testing. These edge effects underline the importance of buffering the core forest areas for protecting existing $H$. antimena populations.

The designation of a protected area provides hope that the largest remaining fragment of deciduous, dry forest in Menabe will be conserved, including strict protection of the core areas of the Kirindy/CFPF and Ambadira forests so crucial for $H$. antimena and sustainable management of the surrounding forest areas. However, the species remains highly threatened because of, among other factors, its restricted range, low reproductive output and the history of widespread and rapid destruction of dry forest in the region. A monitoring scheme has been implemented based on the data collection protocols outlined here, which will be managed in the long-term by the protected area management authority. Increasing the area of suitable habitat outside its current known range, either through forest restoration or regeneration, would improve the chances of long-term survival of $H$. antimena. A habitat suitability model, based on field and satellite data, has now been developed to identify areas of forest for such management that are currently unoccupied but in a state most suitable for H. antimena.

\section{Acknowledgements}

We thank the Ministry of Environment, Waters and Forests of the Government of Madagascar for permission to undertake this research and for the decision to protect the Menabe forests. We are grateful to Conservation International and the US Agency for International Development for funding this field research and our partners Fanamby for their support during the project. We thank the Deutsches Primatenzentrum and Centre de Formation Professionelle Forestière for logistical assistance and technical help. Simone Sommer gave valuable advice on trapping protocols. We are grateful to Len Thomas for helping with the data analysis. Three anonymous reviewers provided useful comments on an earlier draft.

\section{References}

Buckland, S.T., Anderson, D.R., Burnham, K.P., Laake, J.L., Borchers, D.L. \& Thomas, L. (2001) Introduction to Distance Sampling: Estimating Abundance of Biological Populations. Oxford University Press, Oxford, UK.

Cook, J.M., Trevelyan, R., Walls, S.S., Hatcher, M. \& Rakotondraparany, F. (1991) The ecology of Hypogeomys antimena, an endemic Madagascan rodent. Journal of Zoology, 224, 191-200.

Ganzhorn, J.U., Lowry II, P.P, Schatz, G.E. \& Sommer, S. (2001) The biodiversity of Madagascar: one of the world's hottest hotspots on its way out. Oryx, 35, 346-348.

Goodman, S.M. \& Rakotondravony, D. (1996) The Holocene distribution of Hypogeomys (Rodentia: Muridae: Nesomyinae) on
Madagascar. In Biogéographie de Madagascar (ed. W.R. Lourenço), pp. 283-293. ORSTOM, Paris, France.

IUCN (2007) 2007 IUCN Red List of Threatened Species. IUCN, Gland, Switzerland. Http://www.iucnredlist.org [accessed 17 April 2007].

Sмith, A.P. (1997) Deforestation, fragmentation, and reserve design in western Madagascar. In Tropical Forest Remnants: Ecology, Management and Conservation of Fragmented Communities (eds W. Laurance \& O.W. Bierregaard), pp. 415-441. University of Chicago Press, Chicago, USA.

Sommer, S. (1996) Ecology and social structure of Hypogeomys antimena, an endemic rodent of the deciduous dry forest in western Madagascar. In Biogéographie de Madagascar (ed. W.R. Lourenço), pp. 295-302. ORSTOM, Paris, France.

Sommer, S. (1997) Monogamy in Hypogeomys antimena, an endemic rodent of the deciduous dry forest in western Madagascar. Journal of Zoology, 241, 301-314.

Sommer, S. (2001) Reproductive ecology of the endangered monogamous Malagasy giant jumping rat, Hypogeomys antimena. Mammalian Biology, 66, 111-115.

Sommer, S. \& Hommen, U. (2000) Modelling the effects of life history traits and changing ecological conditions on the population dynamics and persistence of the endangered Malagasy giant jumping rat (Hypogeomys antimena). Animal Conservation, 4 , 333-343.

Sommer, S. \& Tichy, H. (1999) MHC-class II polymorphism and paternity in the monogamous Hypogeomys antimena, the endangered, largest Malagasy rodent. Molecular Ecology, 8, 1259-1272.

Sommer, S., Tото Volahy, A. \& Seal, U.S.S. (2002) A population and habitat viability assessment for the highly endangered giant jumping rat (Hypogeomys antimena), the largest extant endemic rodent of Madagascar. Animal Conservation, 5, 263-273.

SorG, J.P. \& Rohner, U. (1996) Climate and tree phenology of the dry deciduous forest of the Kirindy Forest. Primate Report, 46, 57-80.

Thomas, L., Laake, J.L., StrindberG, S., Marques, F.F.C., Buckland, S.T., Borchers, D.L. et al. (2003) Distance 4.0 Release 1. Research Unit for Wildlife Population Assessment, University of St Andrews, UK. Http://www.ruwpa.st-and.ac.uk/ distance/ [accessed 11 June 2008].

Tото Volahy, A. (1999) Contribution à la connâissance du rat sauteur géant de Madagascar, Hypogeomys antimena (A. Grandidier, 1869): essai de détermination des facteurs limitant l'aire de sa répartition dans la forêt dense sèche et caducifoliée de l'ouest. Mémoire DEA Sc., Anthropologie DEA, Université d'Antananarivo, Madagascar.

\section{Biographical sketches}

RICHARD Young specializes in animal abundance estimation techniques, and currently works on a range of projects in Madagascar and the Caribbean researching and monitoring threatened species. Anselme Toto Volahy and Robert Bourou work on a number of endangered species monitoring programmes in western Madagascar. Tim WRIGHT is an animal keeper for Durrell based in Jersey, UK Channel Islands, and conducts field research on the giant jumping rat in Menabe. Richard Lewis is Programme Director of Durrell's conservation programme in Madagascar and Joanna Durbin is former Programme Director. Tim Hounsome has expertise in monitoring animal populations for wildlife conservation and management. JULIA $\mathrm{F}_{\mathrm{A}}$ has undertaken research in a range of conservation biology topics in Europe, Africa and South America and specializes in biodiversity assessment and threatened species management. 